\title{
Micromechanics of slow granular flows
}

\author{
Ravi Gautam ${ }^{1}$ and Prabhu R. Nott ${ }^{1, *}$ \\ ${ }^{1}$ Department of Chemical Engineering, Indian Institute of Science, Bangalore 560012, India
}

\begin{abstract}
It has been contemplated for a long time that dense granular materials flow in a stick-slip manner, and large fluctuations in the stresses are associated with it. However, the particle scale mechanics for this type of macroscopic motion has not been understood so far. We have analyzed the time evolution of contact networks from particle dynamics simulations and found that the rate of change of elastic energy of the packing can distinguish the stick regimes and the slip events. The isostatic criterion (number of contacts for a minimally stable particle) has been used to construct a cascade failure mechanism which reveals that the effect of the random breaking of contacts due to applied shear can be system-spanning for some cases. The size of the cascade failures follows a power law that explains experimentally observed large fluctuations is stresses. We expect that this power law distribution can connect the microstructure of a granular packing to its mechanical response.
\end{abstract}

\section{Introduction}

The abundance of granular matter in nature and its wide applications in industries have inspired a large number of studies to understand and predict its response to applied shear. Dense granular materials exhibit intriguing flow features such as dilatancy [1], shear rate independence of stresses [2] and large fluctuations in the stresses [3]. These features of slow granular flows are known for a long time, but their origins at particle scale are not understood. The unknown mechanism of particle rearrangement in slow granular flows has been the major obstacle in these efforts. It has been hypothesized that when dense granular material is sheared, it flows during quick slip events separated by relatively long stick regimes.

In recent years, granular matter has been studied as a network where the particles are the nodes, and particle contacts are edges (also referred as links or bonds). The extent of these studies are, so far, limited to networks in static packings and evolution in networks when a small strain is applied from the boundaries $[4,5]$. In this study, we have extended the application of network analysis tools to granular flows and analyzed a time series of contact networks in a sheared system. The coordination number (number of contacts) of a particle is an important parameter governing the mechanical stability of that particle. While the packing is sheared, some contacts break and affect the stability of particles associated with broken contacts. We have developed a cascade failure mechanism to estimate the number of particles affected by the breaking of any contact. We found that stick-slip motion is inherent to slow granular flows, and it is the reason behind large fluctuations in stress. The cascade failure mechanism

*e-mail: prnott@iisc.ac.in

A video is available at https://doi.org/10.48448/b3m0-tf07 reveals the micromechanics responsible for macroscopic stick-slip motion.

\section{Cascade Failure Mechanism}

A two-dimensional sheared system of granular material is simulated using the discrete element method (DEM) [6]. The system is considered periodic along the flow direction. The particle positions obtained from the DEM simulations allows us to create a contact network. For a particle to be stable, the net force and torque on the particle must be zero. Therefore, for minimal stability of a particle, the coordination number $z$ must be $2 d$ for frictionless particles, and it must be $d+1$ for frictional particles [7], where $d$ is the dimension of the system. This condition is called isostatic criterion. Some contacts break due to applied shear, resulting in unbalanced force and torque. Suppose the particles associated with broken contacts still have coordination number more than or equal to isostatic requirement. In that case, the forces will rearrange on the existing contacts, and the effect of perturbation will be limited to the particles associated with broken contacts. On the other hand, if some of these particles do not have enough contacts, they will move from their respective positions and perturb all their contacts resulting in percolation of the effect of broken contact to the nearest neighbours. Following the same rules to next nearest neighbours and so on, we get a mechanism for cascade failure in granular packings.

For a hypothetical perturbation that causes the breakage of all contacts of a particle $A$, we can estimate the number of particles which will lose their mechanical stability as per the above-described cascade failure mechanism. Thus, we define a fragile cluster of particle $A$ as the set of particles which will become mechanically unstable if particle $A$ is removed from the packing (figure 1). 


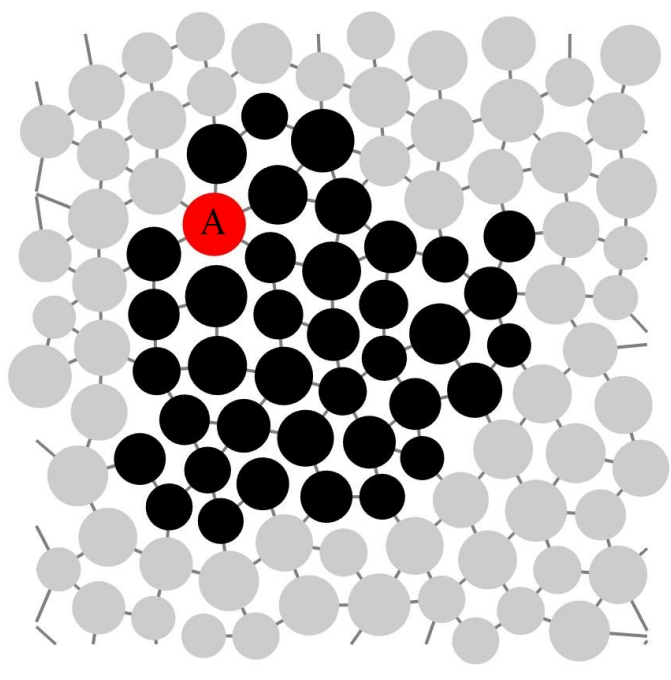

Figure 1. Fragile cluster of Particle $A$.

\section{Results and Discussion}

The contact between particles is approximated by linear spring and dashpot model in the normal and tangential direction; sliding friction is incorporated at all contact points. The time-averaged area fraction of particles is 0.80. We have defined the sum of squares of overlap at all contacts as the elastic energy of the packing. We found that the fluctuations in shear stress and elastic energy are correlated and indicative of the emergence of a macroscopic stick-slip motion where the shear stress and the elastic energy increase monotonically in stick regimes and decrease rapidly in slip events (figure 2). Sudden jumps in kinetic energy at slip events confirm rapid and large rearrangement of the contact network.

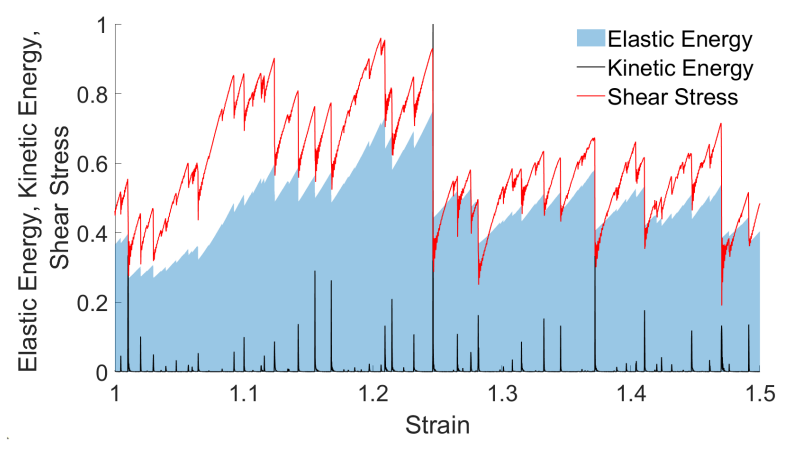

Figure 2. Evidence of macroscopic stick-slip.

We collected a large number of snapshots from the particle dynamics simulation of the sheared granular system. We estimated the fragile cluster size, as defined in section 2 , for each particle at each snapshot. The probability distribution of cluster sizes follows a power law (figure 3) with exponent -2.1. As an alternative to hypothetical perturbation used in defining the fragile cluster, we applied the cascade failure mechanism initiated from the broken contacts between two adjacent snapshots. Thus, the fragile cluster size can be equivalently defined as the fraction of contact network affected by the cascade failure. The distribution of fragile clusters remains similar to the one estimated with hypothetical perturbation. The power-law distribution implies that there is a small but finite probability of system spanning fragile clusters. Simulations at two shear rates, separated by order of magnitude, found that the distribution is independent of shear rate. More importantly, system spanning fragile clusters coincide with the slip events (not shown here).

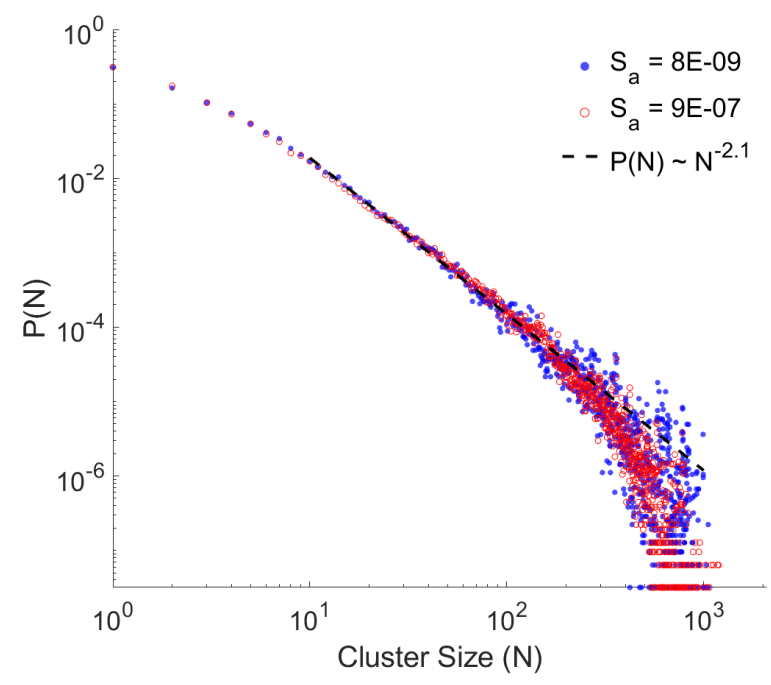

Figure 3. The distribution of fragile cluster sizes at two shear rates separated by order of magnitude. $S_{a}$ refers to Savage number.

These results present a definite picture of the micromechanics of slow granular flows and the emergence of macroscopic stick-slip motion. When the material is sheared, some contacts break randomly and initiate cascade failure. For most of the time, the fragile cluster is small, and the packing can maintain its contact network; thus, the packing is said to be in stick regime. However, when a system spanning fragile cluster forms, the packing goes through large and rapid rearrangement, which is known as a slip event. At each slip event, the packing moves from one jammed state to another. Figure 4 summarizes the micromechanics of slow granular flow.

\begin{tabular}{|c|c|c|}
\hline $\begin{array}{c}\text { Material at a } \\
\text { jammed state }\end{array}$ & $\begin{array}{c}\text { Shear causes } \\
\text { random breaking } \\
\text { of contacts }\end{array}$ & $\begin{array}{c}\text { Broken contacts } \\
\text { initiates cascade } \\
\text { failure }\end{array}$ \\
\hline $\begin{array}{c}\text { Material slips to } \\
\text { another jammed } \\
\text { state }\end{array}$ & $\begin{array}{c}\text { Some fragile } \\
\text { clusters are } \\
\text { system spanning }\end{array}$ & $\begin{array}{c}\text { Fragile cluster size } \\
\text { follow power law } \\
\text { distribution }\end{array}$ \\
\hline
\end{tabular}

Figure 4. Mechanism of granular material moving from one jammed state to another.

These insights provide a phenomenological explanation for rate independence of the stresses in slow granu- 
lar flows. As mentioned earlier, large fluctuations in the stresses are signatures of the underlying stick-slip motion and thus inherent to the flow. Even at different shear rates, the same amount of strain would cause the same numbers of broken contacts; thus, the strain between slip events would be independent of strain rate. The slope of the stress-strain plot in the stick regime depends only on mean coordination number (consequently, the solid fraction). Therefore, at any shear rate within the slow flow regime, the shear stress fluctuates within the same range, independent of the shear rate.

\section{Conclusion}

We have reported a cascade failure mechanism that causes a 'jammed' packing to move in a stick-slip manner. This mechanism reveals that the material slips when a systemspanning fragile cluster forms. We have found that the fragile clusters obey a power-law distribution; thus, there is a non-zero probability of forming a system spanning fragile cluster.

This knowledge about the micromechanics can help us understand the macroscopic behaviour of slow granular flows. We have shown that the fluctuations in shear stress are the result of a macroscopic stick-slip motion. We have also provided phenomenological arguments to explain the rate independence of stress.

\section{References}

1. Reynolds Osborne, London, Edinburgh Dublin Philos. Mag. J. Sci. 20, 469-481 (1885)

2. K. Wieghardt, Annu. Rev. Fluid Mech. 7, 89-114 (1975)

3. B. Miller, C. O’Hern, R.P. Behringer, Phys. Rev. Lett. 77, 3110 (1996)

4. L. Papadopoulos, et al., J. Complex Netw. 6, 485-565 (2018)

5. A.G. Smart, J.M. Ottino, Phys. Rev. E 77, 041307 (2008)

6. P.A. Cundall, O.D.L. Strack, Geotechnique 29, 47-65 (1979)

7. S.F. Edwards, Physica A 249, 226-231 (1998) 